GLOBAL WATER PATHOGEN PROJECT

PART THREE. SPECIFIC EXCRETED PATHOGENS: ENVIRONMENTAL AND EPIDEMIOLOGY ASPECTS

\title{
OVERVIEW OF ISSUES FOR WATER BACTERIAL PATHOGENS
}

\author{
Amy Pruden \\ Virginia Tech \\ Blacksburg, United States \\ Nicholas Ashbolt \\ University of Alberta \\ Jennifer Miller \\ Virginia Tech \\ Blacksburg, United States
}




\section{Copyright:}

\section{cc) (1) (2) \\ BY SA}

This publication is available in Open Access under the Attribution-ShareAlike 3.0 IGO (CC-BY-SA 3.0 IGO) license (http://creativecommons.org/licenses/by-sa/3.0/igo). By using the content of this publication, the users accept to be bound by the terms of use of the UNESCO Open Access Repository (http://www.unesco.org/openaccess/terms-use-ccbysa-en).

\section{Disclaimer:}

The designations employed and the presentation of material throughout this publication do not imply the expression of any opinion whatsoever on the part of UNESCO concerning the legal status of any country, territory, city or area or of its authorities, or concerning the delimitation of its frontiers or boundaries. The ideas and opinions expressed in this publication are those of the authors; they are not necessarily those of UNESCO and do not commit the Organization.

\section{Citation:}

Pruden, A., Ashbolt, N. and Miller, J. 2019. Overview of issues for water bacterial pathogens. In: J.B. Rose and B. Jiménez-Cisneros, (eds) Global Water Pathogen Project.http://www.waterpathogens.org ( A. Pruden, N. Ashbolt and J. Miller (eds) Part 3 Bacteria) http://www.waterpathogens.org/book/overview-of-issues-for-waterbacterial-pathogens Michigan State University, E. Lansing, MI, UNESCO. https://doi.org/10.14321/waterpathogens.20

Acknowledgements: K.R.L. Young, Project Design editor; Website Design (http://www.agroknow.com)

\section{Last published:}




\section{Summary}

\section{An Overview- State of the Knowledge}

The chapters compiled here in Part 3, Section II of the Global Water Pathogens Project provide a detailed overview of the state of the knowledge on key bacterial pathogens relevant to water and sanitation systems. In particular, we provide an overview addressing the last three decades since the Feachem et al. (1983) book was published, highlighting a number of common themes and challenges encountered specifically with each of the bacterial pathogens featured in this section. This brief introductory outlines common themes, with each individual chapter providing detailed information. Note that while there are numerous bacterial pathogens that are relevant to water systems, in general, Part 3 is limited specifically to fecal/enteric bacterial pathogens transmitted via water, i.e., "waterborne" bacterial pathogens. Specifically, each chapter is structured consistently to provide the most current information with respect to pathogen epidemiology, detection methods, environmental occurrence and persistence, and observed removal/inactivation for various sanitation and treatment technologies. Each chapter is based on an exhaustive literature review and provides summary tables, which we hope will be useful to water professionals and researchers around the world in modeling and planning for appropriate water sanitation technologies. A few key points and updates are highlighted in this overview to help guide readers in what to expect in the bacterial pathogen chapters, along with a prospectus of emerging and future challenges with respect to bacterial pathogens in water and sanitation systems.

\subsection{Bacterial Pathogens in Water and Sanitation Systems: Defining Terminology}

In Part I of this book, Chapter 2 "Environmental Aspects and Features of Critical Pathogen Groups," the major features of pathogens and the bacterial members of primary interest for this Section are introduced (Aw, 2018, Environmental Aspects and Features of Critical Pathogen Groups). Decades of public health practice demonstrates that bacterial pathogens of sanitary interest are primarily waterborne (being fecal-oral pathogens spread via water/wastewater/excreta rain-runoff); however, there are also water-wash and water-based bacterial pathogens that include some waterborne members. Important to note is that some enteric bacterial pathogens, such as strains of Aeromonas, pathogenic members of Escherichia coli, Salmonella and Vibrio cholerae, have environmental reservoirs, so as such may not be limited to the traditional fecal-oral pathway of infection. For example, members of the genus Aeromonas are endemic to aquatic environments (wastewater, streams, sediments) and via water exposures some may cause gastroenteritis, wound infections or septicemia. Among Vibrio cholerae, only some strains cause epidemic cholera, which remain a global threat to public health via fecally-contaminated water. However, these bacteria also persist in natural reservoirs associated with free-living protozoa and zooplankton in fresh and brackish waters.

Some non-pathogenic enteric bacteria are typically used as "indicators" of fecal pollution (see Part Two: Indicators and Microbial Source Tracking Markers). Fecal indicator bacteria (FIB) provide a useful monitoring target to assess poor water quality, given standard methods for their enumeration, and safety concerns with direct isolation of human pathogens. However, a point of potential confusion is that among FIB (which are generally non-pathogenic) that typically grow on selective media, human pathogenic strains are sometimes recovered. Examples of FIB that include bacteria of public health concern are pathogenic strains of E. coli, enterococci and Clostridium perfringens. Another issue with the use of FIB is that their response to control technologies, such as disinfection, is not always representative of the target pathogens. For example, FIB may be more sensitive to disinfectants or other removal/inactivation technologies than the target bacterial pathogen of concern and examples where this could lead to underestimating risk are provided in the pathogen-specific chapters. Nonetheless, general reliance on culturable $E$. coli to assess enteric bacterial water quality has proven to be of pragmatic value. As with most indicators, however, limits need to be understood, such as order of magnitude increases in disinfection resistance of some environmental strains of $E$. coli following up-regulation of stress-related genes (Zhi et al., 2019) and actual environmental growth under some conditions (Nanayakkara et al., 2019). The transfer of these attributes to pathogenic bacteria is always a possibility, thus it is important to anticipate such microbial dynamics and reduce environments where antibiotic resistant strains may mix with human bacterial pathogens.

Throughout the bacterial pathogen chapters, the goals were to stay true to the original Feacham text and focus on pathogens of human fecal origin. However, in some cases where such data were sparse, e.g., for environmental occurrence or inactivation data, it was helpful to fill in the gaps with data pertaining to bacteria from animal fecal sources (which is also relevant in settings when human and animal excreta are mixed). For other pathogens, such as Leptospira spp., a major source of concern for humanpathogenic leptospirae is rodent urine, hence animal data are most pertinent. Other relevant zoonotic pathogens described in specific chapters include Campylobacter spp. and Salmonella enterica. A further complication of increasing concern is antimicrobial resistance (AMR) resulting from animal production and companion animal practices (further discussed below). Notably, many zoonotic pathogens may not be pathogenic within the animals that excrete them.

\subsection{Non-Fecal "Water-Based" Pathogens}

While not addressed directly in this section because of the limited the scope to fecal/enteric pathogens, and not to be remiss, other non-fecal pathogens that can be transmitted via water, such as Legionella pneumophila, 
Mycobacterium avium complex, and Pseudomonas aeruginosa must be acknowledged as the important. The term "waterborne" for these pathogens is avoided because this term tends to refer specifically to pathogens of fecal origin and thus instead use the terminology "water-based". Such organisms are also commonly referred to as "opportunistic pathogens," in reference to the fact that they have historically been thought to primarily be of concern to individuals with compromised immune systems, but this term does not recognize that healthy individuals can also be affected and can cause confusion in that some are also enteric pathogens within vulnerable groups. A key distinction between water-based and fecal-based pathogens is that water-based pathogens generally grow and thrive in natural and engineered water systems (Falkinham et al., 2015a; Ashbolt, 2015). This is typically facilitated via growth in biofilms of water treatment and distribution networks. In particular, intracellular growth in amoebae is a common feature among water-based pathogens, which can provide a source of nutrients in oligotrophic drinking water environments and also protection against disinfectants (Thomas and Ashbolt, 2011; Ashbolt, 2015). Another key distinguishing feature among water-based pathogens is that inhalation of aerosols containing the organisms (e.g., Legionella and mycobacteria) and skin colonization (e.g., Pseudomonas aeruginosa) are generally more important than ingestion routes of exposure. This poses new challenges for provision of clean drinking water or non-potable reclaimed wastewater, which traditionally has focused on limiting potential for waterborne pathogen transmission. Not only are these water-based pathogens problematic for drinking water distribution systems and premise plumbing, there is also increasing recognition that water reuse (i.e., recycled treated wastewater) conveyance systems may pose special concerns because of the relatively higher nutrient content of the water, higher temperatures, and greater susceptibility to biofouling (Jjemba et al., 2010). Also, given that the primary route of exposure is aerosol inhalation, more attention should be paid in the future use of such water even for non-potable purposes, such as irrigation (Jjemba et al., 2015). Thus, though not fecal-oral transmitted organisms, the relevance to recycled water distribution does provide a key example of the importance of water-based pathogens in reclaimed wastewater used for sanitation and hygiene applications (Gross et al., 2015). Further, with improved sanitation and decreased fecal-associated disease in developing regions, it can only be expected that disease from water-based pathogens will increase, or at least become more noticeable as seen in developed regions, where they are within the top five communicable disease agents based on disease burden (Cassini et al., 2018; Falkinham et al., 2015b).

\subsection{Methods for Enumerating Bacterial Pathogens- What's New?}

The bacterial pathogen chapters also provide up-to-date information on traditional culture-based and newer molecular-based (i.e., DNA and RNA-targeted) methods for detecting and enumerating bacterial pathogens. Culturebased methods provide an overall advantage in that they directly confirm viability, but there are trade-offs in terms of sub-optimal specificity of media, potential for contamination with non-target strains, and the overall labor and time requirements. Molecular methods generally provide higher specificity and speed than culture and can be formulated to directly target specific virulent strains if desired and thus can be carefully selected depending on the intended target. Overall, a general feature when enumerating bacterial pathogens, whether by culture or molecular detection, is the need to include positive and negative control species to verify specificity.

Traditionally, bacterial pathogens are isolated by culture on various media, which have been formulated and further adapted over the years to improve specificity. Formulating culture media for environmental samples is especially challenging because of the diverse range of background bacteria that can cause false positives or false negatives by out-competing and suppressing growth of the target group. Still, even with highly specific media, it is important to be aware that not all strains within the target bacteria described in this section (Aeromonas, Arcobacter, Campylobacter, pathogenic E. coli/Shigella, Helicobacter pylori, Leptospira, Salmonella enterica, Vibrio cholerae) are actual human pathogens. Further, not all viable (infectious) bacteria are culturable. For example, many enteric/environmental bacteria may form dormant states and lose their culturability when stressed in the environment, a state often termed viable but non-culturable (VBNC), yet can still cause infection. Furthermore, it may take several days to weeks to identify enteric bacteria by culture. This can be a particular disadvantage during outbreak situations.

Overall there has been increasing adoption of molecular methods to improve specificity and identify pathogens within a few hours, such as by using the quantitative polymerase chain reaction (qPCR) and other gene-targeting assays. A downside of PCR-based methods is that cell-free DNA, dead and viable cells are typically not resolved, so depending on the context (such as following disinfection), qPCR enumeration of bacterial pathogens may not be directly informative of viable bacteria. To address this problem, targeting RNA instead of DNA (e.g., via reverse transcription qPCR (RT-qPCR)) can provide an indication of cells that are actually transcribing genes and thus considered viable. The disadvantage of this approach is that RNA is unstable and methods used may lose RNA from environmental samples, leading to underestimations or false negatives. Another approach to the "total count" qPCR problem is pre-treatment of samples with propidium or ethidium monoazide (PMA/EMA), which will generally only allow DNA to amplify from cells with an intact membrane, which prevents entry of these DNA-intercalating dyes prior to extraction, thus improving specificity for 'viable' cell detection by qPCR. However, fine turning of this pretreatment for each target pathogen is generally required to provide useful data, so it is not as straight forward as one may expect (Emerson et al., 2017). 
3.0 Antibiotic Resistance- A New and Critical

\section{Dimension to Combatting Bacterial Pathogens}

One new feature of the bacterial pathogens section is a chapter focused on water and sanitation aspects of antimicrobial resistance as a growing global public health threat. Antibiotics are critical life-saving drugs used to treat bacterial infections, but these drugs are losing their efficacy as bacteria develop the capability to resist antibiotics. Currently it is estimated that there are about 2 million antibiotic resistant infections per year in the U.S., with 18,000 deaths as a result (CDC, 2013). A recent report estimates that globally, death rates due to antimicrobialresistant infections will surpass cancer deaths by the year 2050 , though this estimate does not only include general antibiotic resistance, but also includes general antimicrobial resistance (e.g., to antiparasitics and antivirals) (O’Neil, 2016). Historically, antimicrobial resistance (AMR) has been thought of as a problem primarily in the clinical domain, i.e., with focus on improved hygiene in hospitals, judicious antibiotic use, and new drug discovery. However, recognizing that patients excrete antibiotics and antibiotic resistant bacteria, as do domesticated animals where even larger volumes of antimicrobials are administered, AMR is increasingly being recognized as a one-health issue to control (needing human and animal health as well as environmental domains addressed) (Collignon and McEwen, 2019). As such, antibiotic resistance is a global health challenge made all the more complex as different countries vary in antibiotic use patterns, stringency in prescription requirements (stewardship programs), and sanitation status, while bacteria themselves can freely cross borders with international travel and trade. Hence, the World Health Organization Global Action Plan for Combatting Antibiotic Resistance has recognized water and sanitation as a key linkage and focal point in the battle against the spread of antibiotic resistance, calling upon the global community "to reduce the incidence of infection through effective sanitation, hygiene and infection prevention measures" (WHO, 2015).

Overall, the AMR chapter summarizes the state-of-the science in terms of understanding antimicrobial resistance and the spread of antibiotic-resistance genes (ARGs) as a global water and sanitation challenge (Huijbers et al., 2019). Because ARGs can be shared among bacteria via horizontal gene transfer, particular attention has been paid recently to identifying technologies that effectively remove or attenuate ARGs in bacterial populations. In particular, the tables provide the most comprehensive summary to date of the occurrence of ARGs in various environmental compartments and the effectiveness of treatment technologies for their removal. It is hoped that this information will be a helpful resource to informing identification of effective mitigation technologies against the spread of environmental sources of antibiotic resistance. Particular attention is devoted to sanitation technologies that are promising for developing regions. However, highest priority to control waterborne pathogens should not be compromised due to potential fears in promoting AMR.

\subsection{Relevant Technologies for Sanitation Management}

We aimed to ensure that we emphasized the full range of technologies, ranging from low-cost applications more appropriate to the developing regions all the way to elaborate water treatment more common for non-potable recycled water systems and direct potable reuse. Hence, each bacterial pathogen chapter attempts to address the efficacy of ponds/lagoons, simple anaerobic digesters and composting systems particularly relevant to sanitation in resource-limited environments, through to elaborate, multibarrier treatment trains often used elsewhere. Noting that methods to control bacterial pathogens should also be undertaken with consideration of the other pathogen groups, as addressed in Part 4 of this book.

Here, with the focus is on bacterial pathogens, it is important to note that enteric bacteria are generally the easiest of all pathogen groups to inactivate/remove. However, treatment approaches are typically assessed using traditional FIB, with limited regard to VBNC forms of FIB or actual bacterial pathogens (e.g. VBNC cells are part of the life-cycle of camplyobacters and helicobacters). However, even more problematic is the post-treatment growth of waterborne/water-based bacterial pathogens, including emerging pathogens such as members of Aeromonas, Arcobacter and water-based members not addressed in this book (e.g. legionellae, mycobacteria and Pseudomonas aeruginosa), which is particularly problematic in resource-limited environments that may have no/limited verification monitoring in place.

When compiling bacterial treatment performance by process type, a common problem was the lack of specific pathogen data. Hence, where appropriate the closest relevant FIB for a process was identified, but often "no available data" were observed. Hence, there is a general call out to the research community to prioritize filling these data gaps. For practitioners, the more problematic issues emerge with waterborne pathogens, like some of the Arcobacter spp., that appear to grow in the sewerage infrastructure and likely in other system locations. Also, the water-based pathogens, as discussed above, are increasingly being recognised as critical to control in water holding/conveyance systems where warm stagnant biofilm environments enhance their growth. 


\section{References}

Ashbolt, N.J. (2015). Microbial Contamination of Drinking Water and Human Health from Community Water Systems. Current Environmental Health Reports. 2, pp. 95-106. doi: 10.1007/s40572-014-0037-5.

Aw, T.G. (2018). Environmental Aspects and Features of Critical Pathogen Groups. Global Water Pathogens Project. (Rose, J.B. and Jiménez-Cisneros, B., ed.). Michigan State University and UNESCO. East Lansing, MI, USA. doi: 10.14321/waterpathogens.2.

Cassini, A., Colzani, E., Pini, A., Mangen, M.J., Plass, D., McDonald, S.A. et al. (2018). Impact of infectious diseases on population health using incidence-based disability-adjusted life years (DALYs): results from the Burden of Communicable Diseases in Europe study, European Union and European Economic Area countries, 2009 to 2013. Eurosurveillance. 23(16), doi: 10.2807/1560-7917.ES.2018.23.16.17-00454.

CDC (2013). Antibiotic Resistance Threats in the United States, 2013. U.S. Centers for Disease Control and Prevention.

Collignon, P. and McEWEN, S. (2019). One Health-Its importance in helping to better control antimicrobial resistance. Tropical Medicine and Infectious Disease. 4(1), pp. 22. doi: 10.3390/tropicalmed4010022.

Emerson, J.B., Adams, R.I., Roman, C.M., Brooks, B., Coil, D.A., Dahlhausen, K. et al. (2017). Schrodinger's microbes: Tools for distinguishing the living from the dead in microbial ecosystems. Microbiome. 5(1), pp. 86.

Falkinham III, J.O., Hilborn, E.D., Arduino, M., Pruden, A. and Edwards, M.A. (2015). Epidemiology and Ecology of Opportunistic Premise Plumbing Pathogens: Legionella pneumophila, Mycobacterium avium, and Pseudomonas aeruginosa. Environmental Health Perspectives. 123(8), pp. 749-758. doi: 10.1289/ehp.1408692.

Falkinham, J.O., Pruden, A. and Edwards, M. (2015). Opportunistic Premise Plumbing Pathogens: Increasingly Important Pathogens in Drinking Water. Pathogens. 4(2), pp. 373-386. doi: 10.3390/pathogens4020373.

Gross, A., Maimon, A., Alfiya, Y. and Friedler, E. (2015). Greywater Reuse. CRC Press. Boca Raton, Florida.

Huijbers, P.M.C., Flach, C.F. and Larsson, D.G.J. (2019). A conceptual framework for the environmental surveillance of antibiotics and antibiotic resistance. Environment International. 130, pp. 104880. doi: 10.1016/j.envint.2019.05.074.

Jjemba, P.K., Johnson, W., Bukhari, Z. and LeChevallier, M.W. (2015). Occurrence and control of Legionella in recycled water systems. Pathogens. 4(3), pp. 470-502.

Jjemba, P.K., Weinrich, L.A., Cheng, W., Giraldo, E. and LeChevallier, M.W. (2010). Regrowth of potential opportunistic pathogens and algae in reclaimed-water distribution systems. Applied and Environmental Microbiology. 76, pp. 4169-4178.

Nanayakkara, B.S., O'Brien, C.L. and Gordon, D.M. (2019). Diversity and distribution of Klebsiella capsules in Escherichia coli. Environmental Microbiology Reports. 11(2), pp. 107-117. doi: 10.1111/1758-2229.12710.

O'Neill, J. (2016). The review on antimicrobial resistance. Infection prevention, control and surveillance: limiting the development and spread of drug resistance.

Thomas, J. and Ashbolt, N.J. (2011). Do Free-Living Amoebae in Treated Drinking Water Systems Present an Emerging Health Risk?. Environmental Science and Technology. 45(3), pp. 860-869. doi: 10.1021/es102876y.

WHO (2015). Global action plan on antimicrobial resistance.

Zhi, S., Banting, G., Stothard, P., Ashbolt, N.J., Checkley, S., Meyer, K. et al. (2019). Evidence for the evolution, clonal expansion and global dissemination of water treatment-resistant naturalized strains of Escherichia coli in wastewater. Water Research. 156, pp. 208-222. doi: 10.1016/j.watres.2019.03.024. 